\title{
Prognosis of Single Spinal Metastatic Tumors: Predictive Value of the Spinal Instability Neoplastic Score System for Spinal Adverse Events
}

\author{
Sam Yeol Chang ${ }^{1}$, Jae Hong $\mathrm{Ha}^{1}$, Sang Gyo Seo ${ }^{2}$, Bong-Soon Chang ${ }^{1}$, Choon-Ki Lee ${ }^{1}$, Hyoungmin Kim ${ }^{1}$ \\ ${ }^{1}$ Department of Orthopedic Surgery, Seoul National University Hospital, Seoul, Korea \\ ${ }^{2}$ Department of Orthopedic Surgery, Asan Medical Center, Seoul, Korea
}

Study Design: This was a retrospective cohort study.

Purpose: We evaluated the predictive value of the Spinal Instability Neoplastic Score (SINS) system for spinal adverse events (SAES) in patients with single spinal metastatic tumor.

Overview of Literature: The SINS system was developed to assess spinal instability in patients with single metastatic spinal tumor. However, the system's potential predictive value for SAEs has been partially studied.

Methods: This system was applied to a retrospective cohort of 78 patients with single spinal metastatic tumors. The patients underwent surgical treatment and were postoperatively followed up for at least 2 years or until death. The attribution of each score and total SINS to SAE (vertebral compression fracture [VCF] and spinal cord compression [SCC]) occurrence was assessed using the Cox proportional hazards model.

Results: SAEs occurred on average 7 months after diagnosis of spinal metastasis. The mean survival rate post diagnosis was 43 months. Multivariate analysis using the Cox proportional hazards model revealed that the pain $(p=0.029)$ and spinal alignment $(p=0.001)$ scores were significantly related to VCF occurrence, whereas the pain $(p=0.008)$ and posterolateral involvement $(p=0.009)$ scores were related to SCC occurrence.

Conclusions: Among the components of the SINS system, while pain and spinal alignment showed a significant association with VCF occurrence, pain and posterolateral involvement showed association with SCC occurrence.

Keywords: Neoplasm metastasis; Spinal Instability Neoplastic Score; Compression fractures; Spinal cord compression

\section{Introduction}

The most common site for malignant skeletal metastasis is the spine, and the incidence of spinal metastasis is increasing due to improved survival rates in cancer patients $[1,2]$. Spinal adverse events (SAEs), including vertebral compression fracture (VCF) and spinal cord compression
(SCC), reduce the quality of life in cancer patients with spinal metastasis [3]. To predict such SAEs and ensure appropriate treatment is timely administered, it is important to identify factors related to them.

Spinal instability is an important factor in VCF and SCC occurrence in patients with spinal metastasis [4]. To assess spinal instability and improve communication

Received Dec 5, 2017; Revised Jan 26, 2018; Accepted Mar 4, 2018

Corresponding author: Hyoungmin Kim

Department of Orthopedic Surgery, Seoul National University Hospital, 101 Daehak-ro, Jongno-gu, Seoul 03080, Korea

Tel: +82-2-2072-0357, Fax: +82-2-764-2718, E-mail: hmkim21@gmail.com 
among specialists involved in the treatment of neoplastic spinal disease, the Spinal Oncology Study Group (SOSG) published the Spinal Instability Neoplastic Score (SINS) system in 2010 [5]. A few studies reported moderate to near-perfect reliability of this system [6-10]. Since its introduction, the SINS system has been widely used to classify the degree of spinal instability and facilitate earlier referral of patients requiring surgical intervention for spinal instability $[11,12]$.

However, currently, the predictive value of the SINS system is controversial [13]. Several studies showed conflicting results on investigation of the predictive value of the SINS system for VCF occurrence after radiotherapy [14-21]. In addition, there is lack of evidence of the association between SINS and SCC occurrence, another SAE that markedly influences the quality of life of patients with spinal metastasis. Therefore, further evaluation of the predictive value of the SINS system is warranted. The aim of this study was to validate the ability of the SINS system to predict SAEs (VCF and SCC) in patients requiring surgical intervention.

\section{Materials and Methods}

A consecutive series of patients with single spinal metastatic tumors who underwent surgical treatment for metastasis from January 2008 to December 2009 were retrieved from the database of the picture archiving and communication system and electronic medical record system in the authors' hospital using formulated queries comprising relevant keywords. Patients who were alive at the final follow-up but had a follow-up of $<24$ months were excluded from the study. Because this study was based on a retrospective review of past medical records, it did not require informed consent from the included patients; however, it was approved by the institutional review board of Seoul National University Hospital (IRB approval no., H-1408-134-607).

Patients in whom spinal lesion was the initial presentation of malignancy, diagnosis of spinal metastasis was confirmed by biopsy, whereas those who were treated and followed up after tissue diagnosis of malignancy at the primary site, spinal metastasis was diagnosed by typical magnetic resonance imaging (MRI) radiological findings. The decision to perform surgery was made in a weekly tumor board meeting of various specialists involved in the treatment of spinal metastasis. Multiple factors, including each patient's primary cancer, performance and clinical status, and the predicted survival period, were considered in the decision making. Clinically, patients with severe pain affecting daily activities and ambulation or neurologic deficits caused by spinal metastasis were candidates for surgical treatment. In addition, a single metastatic lesion of the malignancy with favorable prognosis, such as thyroid or breast cancer, was another surgical indication.

We collected demographic data of the patients, including age, sex, and primary site of malignancy. The SINS system was applied to the clinical and radiological status of the patients during diagnosis of spinal metastasis. The survival periods after malignancy diagnosis and spinal metastasis detection were evaluated during follow-up. In addition, the occurrence of two SAEs, VCF and SCC, was evaluated. These SAEs were clinically and radiologically diagnosed using findings of imaging studies, such as simple radiographs, computed tomography, and MRI, and were interpreted by an independent radiologist. However, in patients who previously had vertebral collapse during spinal metastasis detection, further progression of collapse was regarded as an SAE defined as a decrease in the vertebral body height by $>10 \%$ compared with that during previous imaging studies.

For statistical evaluation, we assessed the attribution of each score and total SINS for VCF and SCC occurrence by univariate analysis using the log rank test. To determine the combined effect of factors found to be significant in the univariate analysis, we applied multivariate analysis of the Cox proportional hazards model. Factors with a $p$-value of $<0.10$ in the univariate analysis were used for multivariate analysis, and $p$-values $<0.05$ were considered statistically significant. For statistical analysis, IBM SPSS statistics ver. 23.0 (IBM Corp., Armonk, NY, USA) was used.

\section{Results}

From January 2008 to December 2009, 78 patients were diagnosed with single spinal metastatic tumor in the authors' hospital. Table 1 summarizes the patients' demographic information and application of treatments. The gastrointestinal system was the most common site of primary malignancy, followed by the breast. The survival rate after diagnosis of spinal metastasis differed based on the primary malignancy, i.e., patients with breast cancer survived for on average 6.5 years, whereas those with pancreatic cancer 
Table1. Spinal Instability Neoplastic Score system

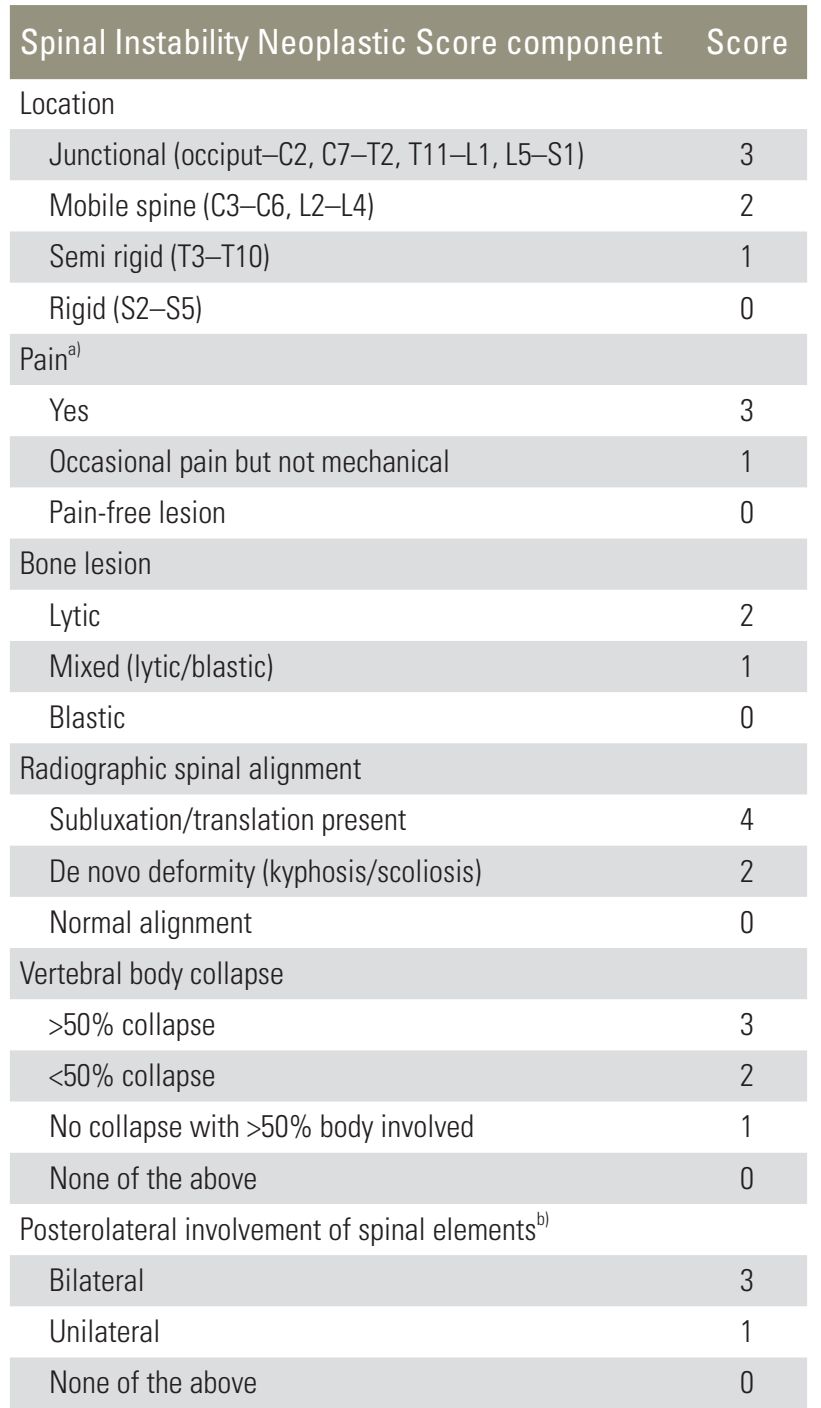

a)Pain improvement with recumbency and/or pain with movement/

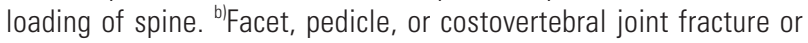
replacement with tumor.

survived for on average only 0.5 years (Table 2).

During diagnosis of spinal metastasis, the distribution of patients into groups according to the SINS system was as follows: stable ( $\mathrm{n}=31$, SINS $<7$ ), impending instability $(\mathrm{n}=44$, SINS $=7-12)$, and instability $(\mathrm{n}=3$, SINS $>12)$. SAEs occurred on average 7 months after diagnosis of spinal metastasis. Of the 78 patients, 24 experienced SAEs during the follow-up, 21 had VCF, 12 had SCC, and nine concomitantly showed both conditions (Table 3 ).

Table 4 summarizes the results of the univariate and multivariate survival analyses using the Cox proportional hazards model. The multivariate analysis showed a significant correlation between the pain $(p=0.008)$ and postero-
Table 2. Demographic data and treatment applications

\begin{tabular}{lc} 
Characteristic & Value \\
Sex & \\
$\quad$ Male & 39 \\
$\quad$ Female & 39 \\
\hline Age (yr) & $55 \pm 13$ \\
\hline Region of spinal metastasis & \\
$\quad$ Cervical & 9 \\
$\quad$ Thoracic & 43 \\
$\quad$ Lumbar & 26 \\
Treatment for spinal metastasis & \\
CTx alone & 26 \\
RTx alone & 20 \\
CTx+RTx & 30 \\
None & 2 \\
\hline
\end{tabular}

Values are presented as number or mean \pm standard deviation.

CTx, chemotherapy; $\mathrm{RTx}$, radiotherapy.

lateral involvement $(p=0.009)$ scores and SCC occurrence (Table 4, Fig. 1) and between the pain $(p=0.029)$ and alignment $(p=0.001)$ scores and VCF occurrence (Table 4 , Fig. 2). The classification into three groups (stability, impending stability, and instability) based on total SINS was not related to SAE occurrence, except for the univariate analysis for VCF occurrence (Table 4). Besides the SINS system, we used the Cox proportional hazards model to evaluate treatment options for spinal metastasis for association with SAEs, but we found no statistical significance ( $p=0.45$ ).

We also evaluated SAE occurrence based on the types of treatment the patients received for spinal metastasis (Table 3). Crosstab analysis of treatment types on SAE occurrence using linear-by-linear association showed no statistical significance $(p=0.141)$. Classification of patients by treatment type into chemoradiotherapy, chemotherapyonly, radiotherapy-only, and no-treatment groups was not associated with VCF and SCC occurrence when assessed by the univariate analysis of the Cox proportional hazards model (VCF, $p=0.974$; SCC, $p=0.874$ ).

\section{Discussion}

SAEs are a major concern for oncologists and other specialists managing patients with spinal metastasis. Emerging therapies such as spine stereotactic body radiotherapy 
Table 3. Survival periods according to primary malignancy

\begin{tabular}{|c|c|c|c|c|}
\hline Organ & Primary cancer & Frequency $(\%)$ & Overall survival (yr) & Survival after spinal metastasis (yr) \\
\hline Breast & Breast & $20(25.6)$ & $8.2 \pm 7.7$ & $6.5 \pm 7.3$ \\
\hline Lung & Lung & $18(23.1)$ & $1.2 \pm 0.9$ & $1.1 \pm 0.8$ \\
\hline \multirow[t]{5}{*}{ Gastrointestinal ( $n=25,32.1 \%)$} & Liver & $8(10.3)$ & $2.9 \pm 1.8$ & $2.7 \pm 1.6$ \\
\hline & Stomach & $5(6.4)$ & $1.9 \pm 2.3$ & $1.8 \pm 2.3$ \\
\hline & Colorectal & $5(6.4)$ & $0.8 \pm 0.5$ & $0.5 \pm 0.5$ \\
\hline & Esophagus & $4(5.1)$ & $1.6 \pm 1.6$ & $1.10 \pm 1.3$ \\
\hline & Pancreas & $3(3.8)$ & $0.5 \pm 0.3$ & $0.3 \pm 0.2$ \\
\hline \multirow[t]{3}{*}{ Genitourinary $(n=10,12.8 \%)$} & Kidney & $6(7.7)$ & $3.0 \pm 2.2$ & $2.2 \pm 1.3$ \\
\hline & Prostate & $2(2.6)$ & $3.4 \pm 2.1$ & $3.3 \pm 2.2$ \\
\hline & Uterus & $2(2.6)$ & $1.5 \pm 1.8$ & $1.1 \pm 1.2$ \\
\hline \multirow[t]{2}{*}{ Endocrine $(n=3,3.9 \%)$} & Thyroid & $2(2.6)$ & $6.4 \pm 1.1$ & $3.7 \pm 2.7$ \\
\hline & Parathyroid & $1(1.3)$ & 2.9 & 2.5 \\
\hline Extremity & Sarcoma & $2(2.6)$ & $1.6 \pm 0.5$ & $1.6 \pm 0.5$ \\
\hline Total & & $78(100.0)$ & $3.6 \pm 5.0$ & $3.0 \pm 4.5$ \\
\hline
\end{tabular}

Values are presented as number or mean \pm standard deviation, unless otherwise stated.

Table 4. Summary of SAE according to treatment

\begin{tabular}{|c|c|c|c|c|c|}
\hline Variable & $\operatorname{VCF}(\mathrm{A})$ & $\operatorname{SCC}(B)$ & $\mathrm{VCF}+\mathrm{SCC}(\mathrm{C})$ & Patients with SAE (A+B-C) & Total no. of patients \\
\hline RTx alone & 6 & 2 & 1 & 7 & 20 \\
\hline CTx alone & 8 & 5 & 4 & 9 & 26 \\
\hline $\mathrm{CTx}+\mathrm{RTx}$ & 6 & 4 & 3 & 7 & 30 \\
\hline None & 1 & 1 & 1 & 1 & 2 \\
\hline Total & 21 & 12 & 9 & 24 & 78 \\
\hline
\end{tabular}

SAE, spinal adverse events; VCF, vertebral compression fracture; SCC, spinal cord compression; RTx, radiotherapy; CTx, chemotherapy.
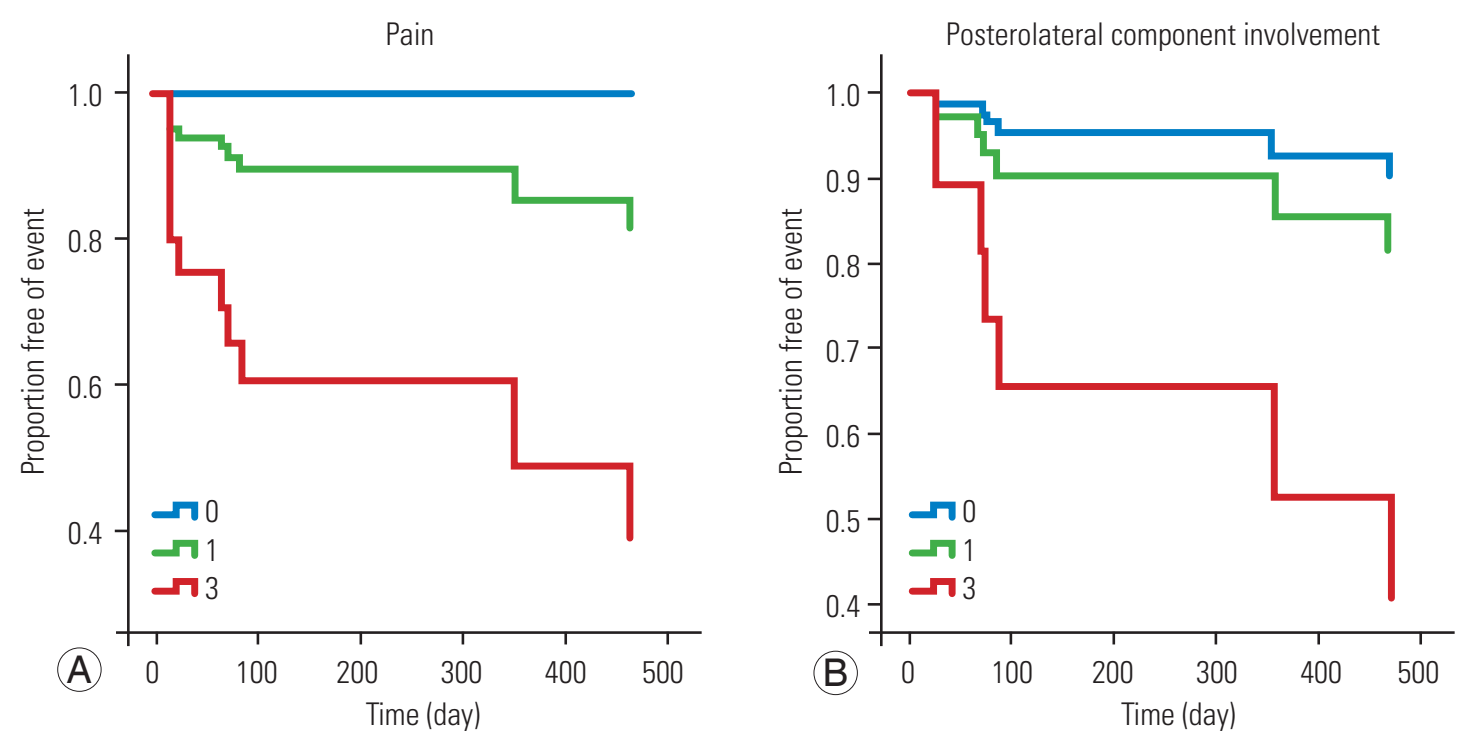

Fig. 1. Survival function graph for spinal cord compression according to the pain score (A) and posterolateral component involvement (B). 

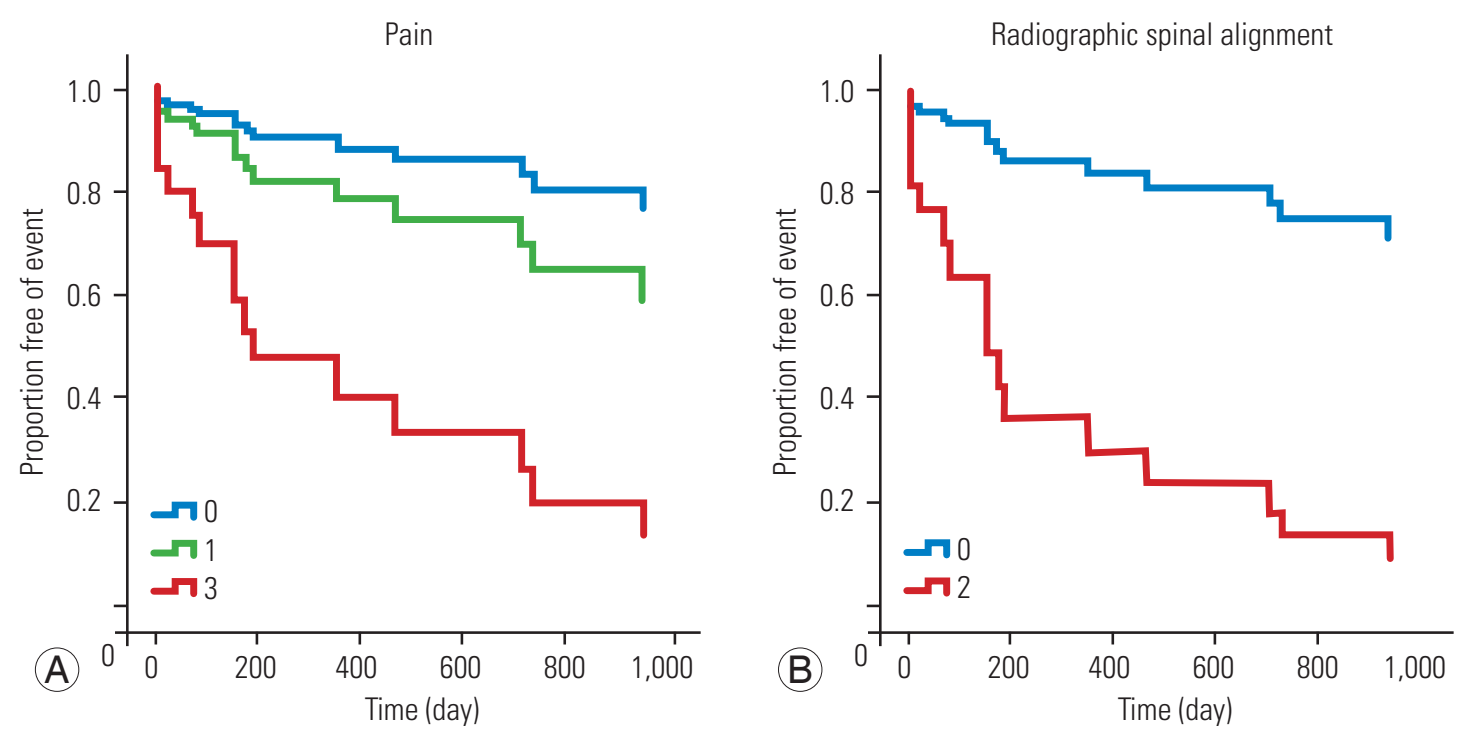

Fig. 2. Survival function graph for vertebral compression according to the pain score (A) and radiographic spinal alignment (B).

(SBRT) have drawn attention to SAE occurrences [22]. More than two decades ago, Mirels [23] proposed some useful criteria for long bone metastasis, which help physicians predict the risk of pathologic fracture in patients with long bone metastasis and provide guidance on whether prophylactic fixation should be used to avoid the debilitating complication of pathologic fracture. However, selecting the most helpful treatment options for patients with spinal metastasis continues to be challenging for spine surgeons and oncologists because of the lack of knowledge to determine the prognosis for patients with spinal metastasis, particularly regarding the risk of VCF and SCC.

There have been many efforts to evaluate and classify the stability of spine with metastatic lesions. Harrington classified spinal metastases into five categories on the basis of bone and neurologic involvement and suggested treatment options for each category [24]. Further, Kostuik et al. [25] proposed a six-column system to develop spinal instability criteria, and Taneichi et al. [26] investigated the risk factors for and probability of vertebral body collapse in the thoracolumbar spine with metastatic lesions. However, none of these classification systems has been completely validated or widely used in a clinical setting, and systemic review of this issue has not reached a definitive conclusion [4].

To assess spinal instability and improve communication between physicians and other specialists involved in the treatment of neoplastic spinal disease, in 2010, the SOSG proposed the SINS system on the basis of questionnaires compiled by a group of experts using the modified Delphi technique [5] (Table 5). A few studies reported moderate to near-perfect reliability of the SINS system [6-10]. Near-perfect intra- and inter-observer reliabilities of this system were also observed when members of the SOSG determined three clinically relevant categories of stability [6]. A few authors reported substantial to excellent interand intra-observer reliability of total SINS $[7,8]$, but some suggested that the degree of reliability depends on the evaluators' experience and varies among different specialties $[9,10]$.

Since its introduction, the SINS system has been widely used to classify the degree of spinal instability and facilitate early referral of patients requiring surgical intervention for spinal instability. In 2013, the American Academy of Orthopedic Surgeons introduced SINS as a classification system of spinal instability in an instructional course lecture for general practitioners [11]. Versteeg et al. [12] found a decrease in total SINS in the cohort after the introduction of the SINS system, explained by increased awareness of spinal instability and earlier referral to a spinal surgeon.

Despite the clinical effect of the SINS system mentioned earlier, its prognostic or predictive value is yet to be validated. Several authors reported the predictive value of the SINS system for VCF occurrence after radiotherapy [1419]. In these studies, the factors found to be associated with VCF occurrence or progression after radiotherapy included a lytic tumor [14,15], malalignment [14,15], preexisting VCF $[15,16,21]$, and total SINS [17-19]. On the 
Table 5. Summary of Cox regression analysis for spinal adverse events

\begin{tabular}{|c|c|c|c|c|}
\hline Spinal adverse events & $\begin{array}{l}\text { Crude HR on univariate } \\
\text { analysis }(95 \% \mathrm{CI})\end{array}$ & $p$-value & $\begin{array}{l}\text { Adjusted HR on multivariate } \\
\text { analysis }(95 \% \mathrm{Cl})\end{array}$ & $p$-value \\
\hline \multicolumn{5}{|l|}{ Vertebral compression fracture } \\
\hline Pain & & 0.014 & & 0.029 \\
\hline 0 & 1.00 & & 1.00 & \\
\hline 1 & $1.99(0.45-16.27)$ & & $0.81(0.08-8.11)$ & \\
\hline 3 & $7.53(1.02-59.94)$ & & $5.12(0.45-33.78)$ & \\
\hline Alignment & & 0.001 & & 0.001 \\
\hline 0 & 1.00 & & 1.00 & \\
\hline 2 & $2.78(1.84-4.20)$ & & $7.69(2.13-15.48)$ & \\
\hline Total SINS score & & 0.032 & & 0.765 \\
\hline$<7$ & 1.00 & & 1.00 & \\
\hline$>7-12$ & 3.93 (1.10-14.03) & & 0.577 (0.09-3.72) & \\
\hline$>12$ & 11.81 (1.09-98.42) & & $0.94(0.04-24.70)$ & \\
\hline \multicolumn{5}{|l|}{ Spinal cord compression } \\
\hline Pain & & 0.006 & & 0.008 \\
\hline 0 & 1.00 & & 1.00 & \\
\hline 1 & $1.35(0.81-3.45)$ & & $1.08(0.54-2.89)$ & \\
\hline 3 & $9.35(1.30-18.23)$ & & $6.24(1.60-24.37)$ & \\
\hline Posterolateral involvement & & 0.001 & & 0.009 \\
\hline 0 & 1.00 & & 1.00 & \\
\hline 1 & $2.02(0.21-19.44)$ & & $1.11(0.11-11.02)$ & \\
\hline 3 & $16.52(2.02-45.40)$ & & 10.89 (1.09-68.59) & \\
\hline Total SINS score & & 0.735 & & 0.812 \\
\hline$<7$ & 1.00 & & 1.00 & \\
\hline$>7-12$ & $>1,000$ & & $>1,000$ & \\
\hline$>12$ & $>1,000$ & & $>1,000$ & \\
\hline
\end{tabular}

$\mathrm{HR}$, hazard ratio; $\mathrm{Cl}$, confidence interval; SINS, spine instability neoplastic score.

other hand, other studies showed no association between SINS and VCF occurrence $[20,21]$. However, other studies investigated prolonged survival after surgical treatment for spinal metastasis but found no prognostic value of SINS [27-29]. However, these studies were limited only to patients receiving a specific treatment such as radiotherapy or surgery and lacked unstable cases (SINS $>12$ ).

Therefore, through the current study, we aimed to elucidate the predictive value of the SINS system for SAE occurrence in patients with spinal metastasis. The composition of patients in our cohort distinguishes us from the previous studies. Only patients with single spinal metastatic tumors were included in the study to control confounding factors. Our cohort showed normal distribution of SINS (Kolmogorov-Smirov test, $p=0.01$ ) with unstable cases (SINS >12) included. In addition, because the cohort comprised patients who underwent different treatments, the results of the study may better represent the natural course of the complete population with spinal metastasis. Furthermore, this study evaluated the association between the SINS system and SCC occurrence, another important SAE, which was not studied in previous reports.

This study showed that VCF and SCC were detected on average 7 months after the detection of spinal metastasis; this result is not much different from the study that reported that skeletal-related events are known to occur on average 6 months after diagnosis of spinal metastasis. The survival rate after diagnosis of spinal metastasis differed depending on the primary tumor. A favorable prognosis of breast and thyroid cancers suggests more aggressive 
treatment in these patients.

Among the six components of the SINS system, three (pain, alignment, and posterolateral involvement) showed a statistically significant relation with SAE occurrence. Considering that the pain score was associated with VCF and SCC occurrence, clinicians who manage cancer patients should pay more attention to patients' axial painrelated complaints, particularly mechanical. Certain radiographic features of spinal metastasis also provide some clues to the possibility of subsequent SAEs and deserve additional attention during follow-up. Previous studies reported the association of spinal malalignment with VCF in patients with spinal metastasis treated by SBRT $[14,15]$. Some studies also showed that metastatic lesions involving a posterolateral spinal element, including the lamina, are prone to causing epidural SCC [30]. On the basis of these results, prophylactic surgical intervention might be considered for patients showing malalignment or posterolateral involvement in imaging studies to prevent SAEs. However, further studies are warranted to draw a conclusion and justify such an approach.

In our study, grouping based on total SINS was not statistically related to SAE occurrence in the Cox proportional hazards model, except for the univariate analysis for VCF occurrence $(p=0.032)$. Previous studies showed conflicting results regarding the association between total SINS and VCF occurrence. Some studies reported statistically significant association between total SINS or grouping of SINS and VCF after SBRT [17-19], whereas others found no significance $[15,20]$. In particular, Sahgal et al. [15] reported that certain components of the SINS system, i.e., a lytic tumor, spinal misalignment, and baseline vertebral collapse, rather than total SINS, were risk factors for VCF occurrence or progression, implying that particular clinical or radiological findings must not be overlooked, even in patients with a low total SINS. However, to include the variety in the disease course across different types of tumors and to provide detailed evidence to predict the risk of SAEs requiring surgical intervention, more prospective studies with a larger volume are necessary.

\section{Conclusions}

This study aimed to validate the ability of the SINS system to predict SAEs requiring surgical intervention. Three components of the SINS system, i.e., pain, alignment, and posterolateral involvement, were associated with SAE oc- currence in the Cox proportional hazards model.

\section{Conflict of Interest}

No potential conflict of interest relevant to this article was reported.

\section{References}

1. Klimo P Jr, Schmidt MH. Surgical management of spinal metastases. Oncologist 2004;9:188-96.

2. American Cancer Society. Cancer facts \& figures 2007. Atlanta (GA): American Cancer Society; 2007.

3. Sutcliffe P, Connock M, Shyangdan D, Court R, Kandala NB, Clarke A. A systematic review of evidence on malignant spinal metastases: natural history and technologies for identifying patients at high risk of vertebral fracture and spinal cord compression. Health Technol Assess 2013;17:1-274.

4. Weber MH, Burch S, Buckley J, et al. Instability and impending instability of the thoracolumbar spine in patients with spinal metastases: a systematic review. Int J Oncol 2011;38:5-12.

5. Fisher CG, DiPaola CP, Ryken TC, et al. A novel classification system for spinal instability in neoplastic disease: an evidence-based approach and expert consensus from the Spine Oncology Study Group. Spine (Phila Pa 1976) 2010;35:E1221-9.

6. Fourney DR, Frangou EM, Ryken TC, et al. Spinal instability neoplastic score: an analysis of reliability and validity from the spine oncology study group. J Clin Oncol 2011;29:3072-7.

7. Campos M, Urrutia J, Zamora T, et al. The Spine Instability Neoplastic Score: an independent reliability and reproducibility analysis. Spine J 2014;14:1466-9.

8. Fisher CG, Versteeg AL, Schouten R, et al. Reliability of the spinal instability neoplastic scale among radiologists: an assessment of instability secondary to spinal metastases. AJR Am J Roentgenol 2014;203:86974.

9. Teixeira WG, Coutinho PR, Marchese LD, et al. Interobserver agreement for the spine instability neoplastic score varies according to the experience of the evaluator. Clinics (Sao Paulo) 2013;68:213-8.

10. Arana E, Kovacs FM, Royuela A, et al. Spine Instability Neoplastic Score: agreement across different medical and surgical specialties. Spine J 2016;16:591- 
9.

11. Quinn RH, Randall RL, Benevenia J, Berven SH, Raskin KA. Contemporary management of metastatic bone disease: tips and tools of the trade for general practitioners. J Bone Joint Surg Am 2013;95:1887-95.

12. Versteeg AL, van der Velden JM, Verkooijen HM, et al. The effect of introducing the Spinal Instability Neoplastic Score in routine clinical practice for patients with spinal metastases. Oncologist 2016;21:95101.

13. Versteeg AL, Verlaan JJ, Sahgal A, et al. The Spinal Instability Neoplastic Score: impact on oncologic decision-making. Spine (Phila Pa 1976) 2016;41 Suppl 20:S231-7.

14. Cunha MV, Al-Omair A, Atenafu EG, et al. Vertebral compression fracture (VCF) after spine stereotactic body radiation therapy (SBRT): analysis of predictive factors. Int J Radiat Oncol Biol Phys 2012;84:e343-9.

15. Sahgal A, Atenafu EG, Chao S, et al. Vertebral compression fracture after spine stereotactic body radiotherapy: a multi-institutional analysis with a focus on radiation dose and the spinal instability neoplastic score. J Clin Oncol 2013;31:3426-31.

16. Thibault I, Al-Omair A, Masucci GL, et al. Spine stereotactic body radiotherapy for renal cell cancer spinal metastases: analysis of outcomes and risk of vertebral compression fracture. J Neurosurg Spine 2014;21:711-8.

17. Finnigan R, Burmeister B, Barry T, et al. Technique and early clinical outcomes for spinal and paraspinal tumours treated with stereotactic body radiotherapy. J Clin Neurosci 2015;22:1258-63.

18. Lam TC, Uno H, Krishnan M, et al. Adverse outcomes after palliative radiation therapy for uncomplicated spine metastases: role of spinal instability and single-fraction radiation therapy. Int J Radiat Oncol Biol Phys 2015;93:373-81.

19. Lee SH, Tatsui CE, Ghia AJ, et al. Can the spinal instability neoplastic score prior to spinal radiosurgery predict compression fractures following stereotactic spinal radiosurgery for metastatic spinal tumor?: a post hoc analysis of prospective phase II singleinstitution trials. J Neurooncol 2016;126:509-17.
20. Sung SH, Chang UK. Evaluation of risk factors for vertebral compression fracture after stereotactic radiosurgery in spinal tumor patients. Korean J Spine 2014;11:103-8.

21. Germano IM, Carai A, Pawha P, Blacksburg S, Lo YC, Green S. Clinical outcome of vertebral compression fracture after single fraction spine radiosurgery for spinal metastases. Clin Exp Metastasis 2016;33:1439.

22. Rose PS, Laufer I, Boland PJ, et al. Risk of fracture after single fraction image-guided intensity-modulated radiation therapy to spinal metastases. J Clin Oncol 2009;27:5075-9.

23. Mirels H. Metastatic disease in long bones: a proposed scoring system for diagnosing impending pathologic fractures. Clin Orthop Relat Res 1989;(249):256-64.

24. Harrington KD. Metastatic disease of the spine. J Bone Joint Surg Am 1986;68:1110-5.

25. Kostuik JP, Errico TJ, Gleason TF, Errico CC. Spinal stabilization of vertebral column tumors. Spine (Phila Pa 1976) 1988;13:250-6.

26. Taneichi H, Kaneda K, Takeda N, Abumi K, Satoh S. Risk factors and probability of vertebral body collapse in metastases of the thoracic and lumbar spine. Spine (Phila Pa 1976) 1997;22:239-45.

27. Zadnik PL, Hwang L, Ju DG, et al. Prolonged survival following aggressive treatment for metastatic breast cancer in the spine. Clin Exp Metastasis 2014;31:4755.

28. Zadnik PL, Goodwin CR, Karami KJ, et al. Outcomes following surgical intervention for impending and gross instability caused by multiple myeloma in the spinal column. J Neurosurg Spine 2015;22:301-9.

29. Ha KY, Kim YH, Ahn JH, Park HY. Factors affecting survival in patients undergoing palliative spine surgery for metastatic lung and hepatocellular cancer: dose the type of surgery influence the surgical results for metastatic spine disease? Clin Orthop Surg 2015;7:344-50.

30. Hamamoto Y, Kataoka M, Senba T, et al. Vertebral metastases with high risk of symptomatic malignant spinal cord compression. Jpn J Clin Oncol 2009;39:431-4. 